\title{
Spilled Gallstones during Laparoscopic Cholecystectomy: Case Series and Review of Literature
}

\author{
May Thu Kyaw ${ }^{1 *}$ and Tai-Ping Lee ${ }^{2}$ \\ ${ }^{1}$ Department of Resident Internal Medicine, Long Island Jewish Forest Hills Hospital, USA \\ ${ }^{2}$ Department of Gastroenterology Internal Medicine, Sandra Atlas Bass Center for Liver Diseases, USA
}

\author{
*Corresponding author: May Thu Kyaw, Department of Resident Internal Medicine, \\ Long Island Jewish Forest Hills Hospital, USA.
}

Received Date: August 12, 2019

Published Date: August 27, 2019

\begin{abstract}
Gallstones can spill into the peritoneal cavity during laparoscopic cholecystectomy. It can cause a silent peritoneal stone and escalate to intraperitoneal complications such as abscess, peritonitis and perforation of intestine from two months up to twenty years. Spilled stone can lead to chronic perihepatic fluid collection, subphrenic abscess and severe life-threatening complications such as sepsis. Every action should be taken to avoid spilling gallstones during the surgery. It warrants high clinical suspicion and requires appropriate interventions. We reviewed complications and management of spilled gallstones after laparoscopic cholecystectomy [1].
\end{abstract}

\section{Case Report}

\section{Case 1}

Patient is a 76-year-old man with past medical history of hypertension, type 2 diabetes mellitus, hyperlipidemia, coronary artery disease, chronic renal insufficiency, alcoholic cirrhosis who was diagnosed with cholelithiasis in 2015. He subsequently developed cholecystitis, which was complicated by choledocholithiasis with cholangitis, requiring ERCP with biliary stent, followed by laparoscopic cholecystectomy. There was no documentation of spilled stones or any other complication during the surgery. Two years after laparoscopic cholecystectomy [2], patient presented with abdominal distention and pain predominantly in the epigastric region, not related to food. Abdomen CT scan showed subphrenic fluid collection extending along right abdominal wall with two to three calcified spilled gallstones. During the hospital course, patient developed fever, leukocytosis and tachycardia. He was diagnosed with sepsis and treated for suspected pneumonia. Patient underwent interventional radiology (IR) guided drainage for multiloculated complex hepatic fluid. Eight hundred milliliters of greenish viscous fluid were aspirated [3]. Culture from intraperitoneal aspiration is negative. IR failed to remove gallstone after dilating the access. Patient underwent an extensive peritoneal washout and perihepatic fluid collection resolved Figure 1.

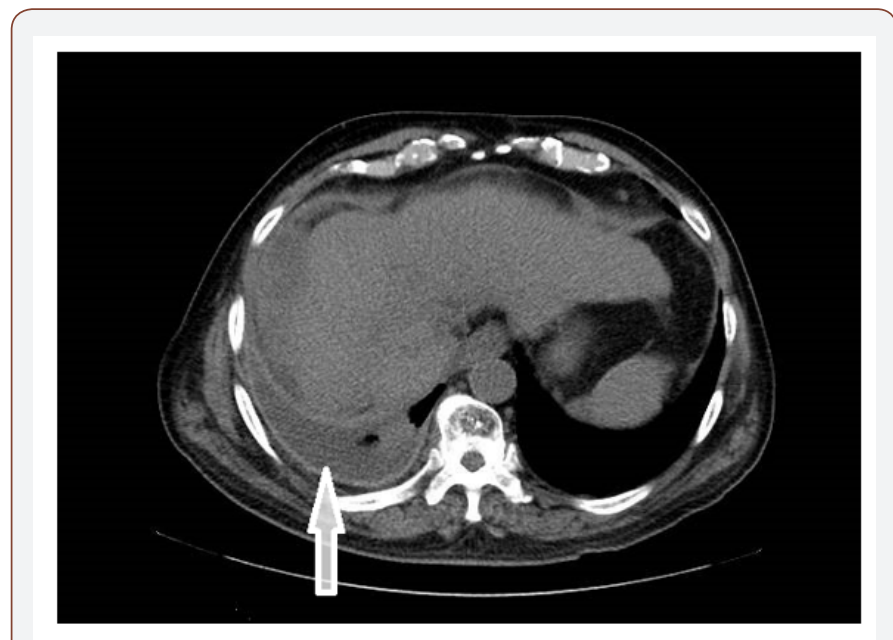

Figure 1: 76 years old Male with Subphrenic Heterogenous Fluid Collection extending Right Abdominal Wall.

\section{Case 2}

A 72-year-old woman with history of laparoscopic cholecystectomy 2 years ago presented with right sided abdominal pain, vomiting and fever. Patient was known to have spilled gallstone in peritoneum post cholecystectomy, require intraperitoneal drain 
[4]. Abdominal CT scan showed three collections; a 3.2x8.3x8.9 cm multiloculated collection tracking around the right posterolateral aspect of the chest and abdominal wall posterior to the area of liver, at the level of the 10th rib with a small calcified focus suspicious for gallstone, an additional area of soft tissue attenuation adjacent to the collection, lateral to the ascending colon representing phlegmonous change, and a $2 \times 3 \times 5.9 \mathrm{~cm}$ multiloculated collection within the anterolateral right abdominal wall near the location of the previous surgical drain. At the same time, patient developed a strangulated ventral hernia requiring emergency open reduction surgery. She was started on antibiotics for abdominal abscess and abdominal drainage. Subsequently, a small gallstone appearing as clip in the center of the abscess cavity was removed. Follow up CT abdomen showed decrease in the size of the abscess cavity. Patient clinically improved and abscess eventually resolved Figure 2.

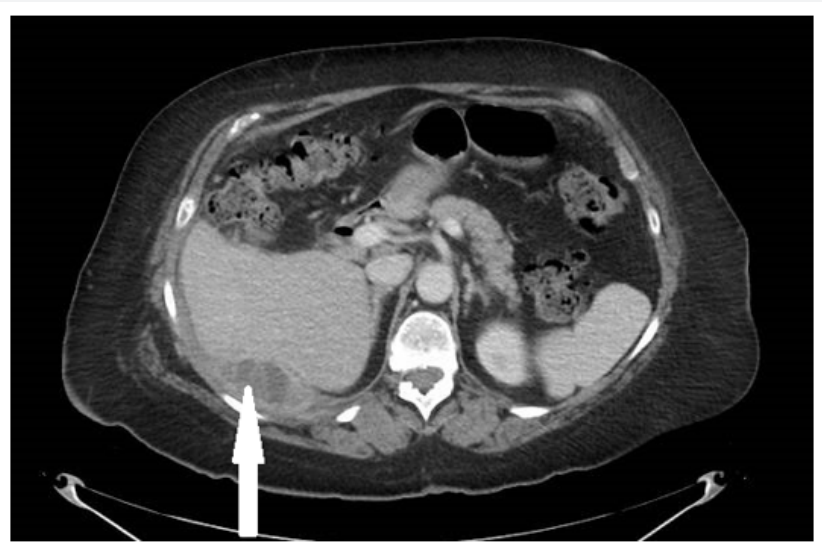

Figure 2: 72 years old Female with Stable Multiloculated Abscess involving Right Abdominal Wall.

Case 3

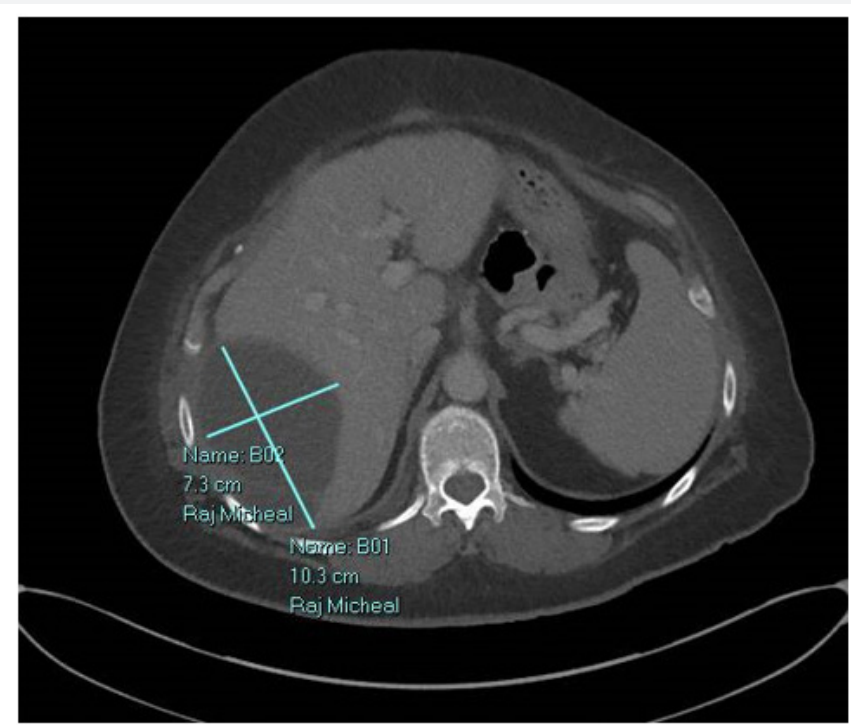

Figure 3: Complex Right Subscapular Hepatic Collection.

54 year-old-woman with history of gastric bypass surgery and laparoscopic cholecystectomy about 6 years ago, presented with perihepatic fluid collection from spilled gallstone $[5,6]$. Patient developed chronic supraumbilical fullness and abdominal distention and found to have increased right subcapsular perihepatic fluid collection on serial abdominal CT scan. Patient underwent IR guided drainage for perihepatic fluid collection, but the fluid collection recurred to $6.1 \times 3.4 \times 6.6 \mathrm{~cm}$ after drainage was removed. She was treated with broad spectrum antibiotics. Patient underwent repeat laparoscopic hepatic infected fluid drainage [7]. Patient was treated with Ciprofloxacin and Metronidazole for Penicillin sensitive E. coli in infected fluid. Follow up abdominal CT abdomen three months later showed decrease in size of the cavity to $4.3 \times 1.6 \times 5.0 \mathrm{~cm}$ after percutaneous drainage removal. Patient is under surveillance for recurrence of perihepatic fluid and peritoneal wash out is planned Figure 3.

\section{Discussion}

Traditional open cholecystectomy was replaced by laparoscopic cholecystectomy for symptomatic gall stones due to shorter dursation of hospital stay, fewer complications, faster recovery time, and less pain [8]. 1,2 Bile duct injury, gall bladder perforation, spillage of gallstone are more common with laparoscopic procedure. Spillage of gallstones occur in $25-30 \%$ cases after laparoscopic cholecystectomy.3 Twenty percentage of gallstone spillage was unnoticed by the surgeon although long term consequences from spilled gallstone are extremely rare.2 The composition of the stones and the component of the bile are the main indicators for future complications of symptomatic spilled gallstones.4 As per Aytekin et al experimental studies with a rat model shows spilled gallstones increase the risk for postoperative adhesion.5 Patients with acute cholecystitis have increased incidence of spillage of infected gallstones and also have higher rate of complications. 6 The onset of the complication varies from a few months to 20 years. 7 Intraabdominal fluid collection or abscess, abdominal wall abscess and fistula formation are seen in descending order. The most common intra-abdominal fluid or abscess is perihepatic in subphrenic area as in our patient. 8 Every attempt should be made to retrieve stone lost during laparoscopic cholecystectomy due to increased risk of complications in the future.9 However, not enough data has been reported whether dropped gallstones should be chased during procedure by transforming into open laparotomy. The best option is the primary prevention which include avoiding the gall bladder perforation, using specimen bag to avoid gallstones leakage, and detail documentation of the spilled stones.10 Every attempt should be made during the procedure to retrieve the stones dropped into the abdominal cavity [9]. Conversion to open procedure to retrieve the stones as secondary prevention is supported when there is infected stones, multiple stones, or suspicion of bacterobilia. Management of abscess from dropped stone depends on the location and size of the abscess. Intravenous antibiotics, saline irrigation and percutaneous IR guided drainage are the treatment of choice for small subphrenic abscess. Calculi in deep seated intraperitoneal pouch which are difficult to access may need open procedure if patient has persistent symptoms [10]. Ultimately, all the stones should be removed while draining the abscess to prevent further irritation and recurrent abscess. 


\section{Conclusion}

Complications arising from spilled gallstones are uncommon. They could take months to years to occur and can sometimes lead to severe comorbidities such as adhesion with small intestinal obstruction, abscess formation and sepsis. Therefore, high suspicion for diagnosis is necessary. Every possible effort should be made to prevent and retrieve the infected or noninfected spilled gallstones during the procedure due to potential complications. However, it is controversial whether it should be converted to open surgery to retrieve a spilled gallstone. Irrigation of abdominal cavity after gall bladder perforation as primary prevention and percutaneous drainage of intra-abdominal abscess are the main stay of management.

\section{Acknowledgement}

None.

\section{Conflict of Interest}

No conflict of interest.

\section{References}

1. Singh K, Wang ML, Ofori E, Widmann W, Alemi A, et al. (2012) Gallstone abscess as a result of dropped gallstones during laparoscopic cholecystectomy. Int J Surg Case Rep 3(12): 611-613.
2. Khalid M, Rashid M (2009) Gallstone abscess: a delayed complication of spilled gallstone after laparoscopic cholecystectomy. Emerg Radiol 16(3): 227-229.

3. Ragozzino A, Puglia M, Romano F, Imbriaco M (2016) Intra-Hepatic Spillage of Gallstones as a Late Complication of Laparoscopic Cholecystectomy: MR Imaging Findings. Polish J Radiol 81: 322-324.

4. Rice DC, Memon MA, Jamison RL, Agnessi T, Ilstrup D, et al. (1997) Longterm consequences of intraoperative spillage of bile and gallstones during laparoscopic cholecystectomy. J Gastrointest Surg 1(1): 85-91.

5. Zehetner J, Shamiyeh A, Wayand W (2007) Lost gallstones in laparoscopic cholecystectomy: all possible complications. Am J Surg 193(1): 73-78.

6. Schäfer M, Suter C, Klaiber C, Wehrli H, Frei E, et al. (1998) Spilled gallstones after laparoscopic cholecystectomy. A relevant problem? A retrospective analysis of 10,174 laparoscopic cholecystectomies. Surg Endosc 12(4): 305-309.

7. Sathesh-Kumar T, Saklani AP, Vinayagam R, Blackett RL (2004) Spilled gall stones during laparoscopic cholecystectomy: A review of the literature. Postgrad Med J 80(940): 77-79.

8. Brockmann JG, Kocher T, Senninger NJ, Schürmann GM (2002) Complications due to gallstones lost during laparoscopic cholecystectomy. Surg Endosc Other Interv Tech 16(8): 1226-1232.

9. Johnston S, O Malley K, McEntee G, Grace P, Smyth E, et al. (1994) The need to retrieve the dropped stone during laparoscopic cholecystectomy. Am J Surg 167(6): 608-610.

10. Horton M, Florence MG (1998) Unusual abscess patterns following dropped gallstones during laparoscopic cholecystectomy. Am J Surg 175(5): 375-379. 